\title{
Trefoil Factor 3 Predicts Incident Chronic Kidney Disease: A Case-Control Study Nested within the Atherosclerosis Risk in Communities (ARIC) Study
}

\author{
Brad C. Astor ${ }^{a-c}$ Anna Köttgen ${ }^{b, d}$ Shih-Jen Hwang ${ }^{e}$ Nrupen A. Bhavsara,b \\ Caroline S. Fox ${ }^{\mathrm{e}, \mathrm{f}}$ Josef Coresh ${ }^{\mathrm{a}-\mathrm{c}, \mathrm{g}}$ \\ ${ }^{a}$ Welch Center for Prevention, Epidemiology and Clinical Research, Johns Hopkins University, ${ }^{\text {b }}$ Department of \\ Epidemiology, Johns Hopkins Bloomberg School of Public Health, and ' Department of Medicine, Johns Hopkins \\ School of Medicine, Baltimore, Md., USA; ${ }^{d}$ Renal Division, University Hospital Freiburg, Freiburg, Germany; \\ eNational Heart, Lung and Blood Institute's Framingham Heart Study and the Center for Population Studies, \\ Bethesda, Md. and Framingham, Mass., ${ }^{\mathrm{f}}$ Division of Endocrinology, Hypertension, and Metabolism, Brigham and \\ Women's Hospital and Harvard Medical School, Boston, Mass., and ${ }^{9}$ Department of Biostatistics, Johns Hopkins \\ Bloomberg School of Public Health, Baltimore, Md., USA
}

\section{Key Words}

Kidney disease $\cdot$ Tubulointerstitual disease $\cdot$ Biomarkers

\begin{abstract}
Background: Early detection of individuals at high risk for chronic kidney disease (CKD) may aid prevention. Urinary levels of trefoil factor 3 (TFF3) are associated with acute kidney injury in animal models, but the association of TFF3 levels with incident CKD in humans is unknown. Methods: We conducted a case-control study nested within the Atherosclerosis Risk in Communities (ARIC) Study and the ARIC Carotid MRI Study to determine whether urinary TFF3 levels predict incident CKD over 8.6 years of follow-up. A total of 143 participants with incident CKD (eGFR decreasing by $\geq 25 \%$ to $<60 \mathrm{ml} / \mathrm{min} / 1.73 \mathrm{~m}^{2}$ ) were matched on age, sex and race to 143 non-cases. Results: Higher TFF3 levels at baseline were strongly associated with Black race, diabetes (both $p=0.002)$, and antihypertensive medication use $(p=$ 0.02). Compared to participants with TFF3 levels in the lowest quartile, the odds ratio (OR) of incident CKD was 1.84 ( $95 \%$ confidence interval $(\mathrm{Cl}): 0.80,4.22$ ) for individuals with TFF3 levels in the second quartile, $2.43(95 \% \mathrm{Cl}: 1.06,5.53)$ for
\end{abstract}

the third quartile, and $2.77(95 \% \mathrm{Cl}: 1.22,6.28)$ for the fourth quartile ( $p$ trend $=0.02$ ). Adjustment for covariates, including urinary albumin: creatinine ratio, did not markedly change the associations. Twofold higher TFF3 levels were strongly associated with incident CKD after adjustment for CKD risk factors (adjusted OR $=1.35 ; 95 \% \mathrm{Cl}: 1.11,1.64$ ). Conclusions: Higher urinary TFF3 levels may indicate ongoing repair of damage in the kidney. Additional studies are needed to confirm whether TFF3 can be useful as a marker of increased risk for CKD.

Copyright $\odot 2011$ S. Karger AG, Basel

\section{Introduction}

Chronic kidney disease (CKD) is associated with an elevated risk of cardiovascular disease, end-stage renal disease, and mortality, even at moderately decreased levels of glomerular filtration rate (GFR) [1-3]. Recent research has focused on factors associated with the progression of existing CKD [4-7], with relatively few studies investigating predictors of incident CKD [8-10]. Early detection of individuals at increased risk of developing

\section{KARGER}

(C) 2011 S. Karger AG, Basel

Fax +41613061234 E-Mail karger@karger.ch www.karger.com www.karger.com/ajn
Brad Astor, $\mathrm{PhD}, \mathrm{MPH}$

Departments of Epidemiology and Medicine, Johns Hopkins University

2024 East Monument Street, Suite 2-600

Baltimore, MD 21287 (USA)

Tel. +1 410502 2779, E-Mail bastor@jhsph.edu 
CKD may allow timely initiation of measures to prevent CKD and its complications [11]. Current measures of kidney function (e.g., GFR estimated from serum creatinine) and kidney damage (e.g., urinary excretion of albumin) used to diagnose CKD are imprecise and may be insensitive to mildly impaired kidney function or damage $[7,12]$. The identification of new markers may allow improved prediction of CKD risk, better targeting of patients for risk-reducing therapies, and potentially a better understanding of the pathophysiologic processes leading to CKD.

In 2008, the US Food and Drug Administration (FDA) and its European counterpart, the European Medicines Evaluation Agency (EMEA), through the Critical Path Initiative [13], jointly accepted seven urinary biomarkers for use in non-clinical drug development as indicators of acute drug-induced kidney toxicity. Among these biomarkers is trefoil factor 3 (TFF3), also known as intestinal trefoil factor (ITF), a peptide which is expressed in goblet cells of the intestines, colon and kidney [14]. Although the function of TFF3 has not been fully characterized, the members of the trefoil family play an integral role in the protection and repair of the gastrointestinal mucosa. Urinary levels of TFF3 are associated with acute kidney injury in animal models [15]. It is unknown, however, whether urinary levels are associated with incident CKD in humans.

We tested whether urinary levels of TFF3 are associated with incident CKD among individuals without decreased GFR or albuminuria in a case-control study nested within the Atherosclerosis Risk in Communities (ARIC) Study and the ARIC Carotid MRI Study.

\section{Methods}

\section{ARIC Study and ARIC Carotid MRI Study}

The ARIC Study is a prospective biracial observational cohort of 15,792 individuals, recruited from a probability sample of four US communities (Forsyth County, N.C.; Jackson, Miss.; suburban Minneapolis, Minn., and Washington County, Md., USA). Participants took part in clinic examinations, starting with a baseline visit during 1987-89, at which time participants were 45-64 years of age, and including three follow-up examinations at approximately 3 -year intervals, during which carotid artery intima-media thickness (IMT) was measured using B-mode ultrasound [16]. We used ARIC Exam 4, conducted in 1996-98, as the baseline visit for this case-control analysis, as this was the first visit at which urine specimens were collected. A total of 11,656 participants attended ARIC Exam 4.

The ARIC Carotid MRI Study examination served as the follow-up visit for this case-control analysis. Participants for the ARIC Carotid MRI Study were selected in 2004-05 from the sur- viving ARIC Study participants under a stratified sampling plan, based on the most recent IMT and field center [17]. The goal was to recruit 1,200 participants with high values of maximum carotid artery IMT at their most recent ultrasound examination (ARIC Exam 3 or 4, 1993-95 or 1996-98) and 800 individuals randomly sampled from the remaining eligible participants. A total of 4,306 persons were invited: 1,403 refused, 837 were ineligible, and 2,066 participated.

\section{Selection of Cases and Controls}

Cases and controls were selected from ARIC participants with data on serum creatinine at both ARIC Exam 4 (baseline) and the ARIC Carotid MRI Study visit (follow-up). Participants with an estimated GFR (eGFR) $<60 \mathrm{ml} / \mathrm{min} / 1.73 \mathrm{~m}^{2}$ or a urinary albumin:creatinine ratio $(A C R) \geq 30 \mathrm{mg} / \mathrm{g}$ at baseline were excluded. Cases of incident CKD were identified as those participants with an eGFR $<60 \mathrm{ml} / \mathrm{min} / 1.73 \mathrm{~m}^{2}$ at follow-up and a decrease in eGFR from baseline to follow-up of $\geq 25 \%$ [18]. Controls were frequency matched to cases by race, sex, and 5-year age categories.

\section{Data Collection}

Measurement of TFF3 was conducted using an immunoassay on a bead Luminex platform (Rules Based Medicine, Austin, Tex., USA) after centrifugation at 4,000 rpm for $5 \mathrm{~min}$. The assay for TFF3 has an interassay coefficient of variation of $12 \%$ at a mean concentration of $0.3 \mu \mathrm{g} / \mathrm{ml}$ and $13 \%$ at a mean concentration of $12 \mu \mathrm{g} / \mathrm{ml}$. Eight participants (5 cases and 3 controls) had TFF3 levels below the limit of detection and these values were set 0.064 $\mu \mathrm{g} / \mathrm{ml}$, the lowest value measured among ARIC participants.

A modified kinetic Jaffé method was used to measure serum creatinine and the abbreviated Modification of Diet in Renal Disease (MDRD) Study equation was used to estimate GFR [19, 20]. The creatinine concentration for ARIC Visit 4 values was corrected for interlaboratory differences and indirectly calibrated to the Cleveland Clinic measurement by the addition of $0.18 \mathrm{mg} / \mathrm{dl}$ $[19,20]$. Creatinine concentrations measured at the ARIC Carotid MRI Study visit were IDMS traceable. Spot urine samples were collected and stored at $-70^{\circ} \mathrm{C}$. Urinary albumin levels were measured by nephelometry and urinary creatinine levels were measured using the Jaffé method.

Systolic and diastolic blood pressures were measured following $5 \mathrm{~min}$ of rest. Three seated measurements were taken by certified technicians using a random-zero sphygmomanometer. The average of the second and third readings was recorded. Diabetes mellitus was defined as a fasting glucose of $\geq 126 \mathrm{mg} / \mathrm{dl}$, nonfasting glucose of $\geq 200 \mathrm{mg} / \mathrm{dl}$, use of oral hypoglycemic medication or insulin, or self-reported physician diagnosis of diabetes mellitus. Plasma cholesterol, triglycerides and high-density lipoprotein cholesterol were determined using enzymatic methods, and low-density lipoprotein cholesterol was calculated using the Friedewald equation [21]. Body mass index (BMI) was calculated as weight $[\mathrm{kg}] /$ height $[\mathrm{m}]^{2}$. Current smoking status and race were defined by self-report.

\section{Statistical Analyses}

Levels of TFF3 and covariates were compared between cases and controls with t tests or $\chi^{2}$ tests, as appropriate. Pearson correlation coefficients were calculated for TFF3 with the other covariates. The concentrations of TFF3 and ACR were skewed and were therefore natural log-transformed to achieve approximate 
Table 1. Characteristics at baseline among participants in the ARIC Study, by case status

\begin{tabular}{|c|c|c|c|}
\hline & Control $(n=143)$ & Case $(n=143)$ & $\mathrm{p}$ value \\
\hline Age, years ${ }^{\mathrm{a}}$ & $64.6(5.7)$ & $64.7(5.5)$ & - \\
\hline Female, $\%^{a}$ & 53.8 & 53.8 & - \\
\hline Black, $\%^{\mathrm{a}}$ & 22.4 & 22.4 & - \\
\hline Diabetes, $\%$ & 14.7 & 18.2 & 0.43 \\
\hline Systolic blood pressure, $\mathrm{mm} \mathrm{Hg}$ & $127.7(18.1)$ & $134.2(19.6)$ & 0.003 \\
\hline Hypertension medication use, $\%$ & 37.1 & 39.9 & 0.63 \\
\hline BMI & $27.6(4.7)$ & $29.1(5.4)$ & 0.02 \\
\hline Current smoking, $\%$ & 14.0 & 12.7 & 0.75 \\
\hline High-density lipoprotein cholesterol, mg/dl & $51.6(18.3)$ & $49.9(18.0)$ & 0.43 \\
\hline Triglycerides, $\mathrm{mg} / \mathrm{dl}$ & $130.5(68.5)$ & $153.1(91.5)$ & 0.02 \\
\hline Urinary ACR, mg/g & $3.1[16.6,6.7]$ & $3.4[1.3,7.7]$ & 0.62 \\
\hline $\mathrm{TFF} 3, \mu \mathrm{g} / \mathrm{ml}$ & $0.5[0.2,1.0]$ & $0.7[0.4,1.2]$ & 0.005 \\
\hline Baseline eGFR, $\mathrm{ml} / \mathrm{min} / 1.73 \mathrm{~m}^{2}$ & $83.2(15.7)$ & $81.6(14.0)$ & 0.36 \\
\hline Follow-up eGFR, $\mathrm{ml} / \mathrm{min} / 1.73 \mathrm{~m}^{2}$ & $80.8(19.3)$ & $48.4(7.6)$ & - \\
\hline Change in eGFR, $\mathrm{ml} / \mathrm{min} / 1.73 \mathrm{~m}^{2}$ & $-2.4(18.4)$ & $-33.2(14.9)$ & - \\
\hline
\end{tabular}

Continuous characteristics are presented as mean (SD) except urinary ACR and TFF3, which are presented as median [25th, 75th percentile]. ${ }^{a}$ Matching characteristics.

normality for continuous analyses. The association between the log-transformed values of TFF3 and case status was examined continuously and by quartiles. A logistic regression cubic spline model with knots at the 25,50 and 75 th percentiles was used to assess the continuous relationship between TFF3 and incident CKD. This model was adjusted for age (55 years) and sex (female) for graphical presentation. Conditional logistic regression was used to calculate unadjusted and adjusted odds ratios (OR) associated with twofold higher TFF3. Adjustment was performed for systolic blood pressure, use of antihypertensive medication, diabetes, current smoking, BMI, high density lipoprotein cholesterol, triglycerides, eGFR, log of ACR, and the ARIC Carotid MRI Study sampling variables of center and IMT strata. Deviation from nonlinearity was assessed with likelihood ratio tests comparing a linear model to models including $2 \mathrm{nd}, 3 \mathrm{rd}$, 4 th and 5 th order polynomials. Analyses were repeated after stratifying by sex, race and diabetes. Analyses also were repeated for the ratio of urinary TFF3 to urinary creatinine. Statistical analyses were conducted using STATA, version 10 (StataCorp, College Station, Tex., USA).

\section{Results}

\section{Baseline Study Characteristics of ARIC Study Participants}

A total of 143 cases were identified and matched to 143 controls. The mean age of the participants was 64.7 years at baseline (table 1). Approximately 53\% were female and $22 \%$ were Black. Cases had a higher mean systolic blood pressure, BMI and triglycerides than controls. The prevalence of diabetes $(18.4 \%$ in cases, $14.7 \%$ in controls; $\mathrm{p}=$
0.43 ) and urinary ACR levels (median $=3.4 \mathrm{mg} / \mathrm{g}$ in cases and $3.1 \mathrm{mg} / \mathrm{g}$ in controls; $\mathrm{p}=0.62$ ) at baseline did not differ significantly by case status. The mean eGFR at baseline also did not differ significantly between cases $(81.6$ $\left.\mathrm{ml} / \mathrm{min} / 1.73 \mathrm{~m}^{2}\right)$ and controls $\left(83.2 \mathrm{ml} / \mathrm{min} / 1.73 \mathrm{~m}^{2}\right)$.

The median TFF3 level was $0.55 \mu \mathrm{g} / \mathrm{ml}$ (interquartile range: $0.27-1.10)$. Higher TFF3 levels were strongly associated with Black race and diabetes (both $\mathrm{p}=0.002$; table 2). Higher TFF3 levels also were associated with antihypertensive medication use $(\mathrm{p}=0.02)$. Participants in the lowest TFF3 quartile were much more likely to be female than those with higher TFF3 levels. Higher TFF3 quartiles remained associated with each of these variables after adjustment for the others (all $\mathrm{p} \leq 0.05$ ).

\section{Association of TFF3 with Incident CKD Case Status in the ARIC Study}

Median TFF3 levels were significantly higher in cases $(0.7 \mu \mathrm{g} / \mathrm{ml})$ than in controls $(0.5 \mu \mathrm{g} / \mathrm{ml} ; \mathrm{p}=0.005)$ (fig. 1$)$. Compared to participants with TFF3 levels in the lowest quartile, the OD of incident CKD was 1.84 (95\% confidence interval (CI): $0.80,4.22)$ for individuals with TFF3 levels in the second quartile, 2.43 (95\% CI: 1.06, 5.53) for the third quartile, and 2.77 (95\% CI: 1.22, 6.28) for the fourth quartile ( $\mathrm{p}$ trend $=0.02$; table 3 ). Adjustment for covariates strengthened these associations slightly ( $p$ trend $=0.01$ ). Further adjustment for urinary ACR did not markedly change the associations ( $p$ trend $=0.02$ ). 
Table 2. Characteristics of participants in the ARIC Study, by TFF3 quartile

\begin{tabular}{|c|c|c|c|c|c|}
\hline & \multicolumn{4}{|l|}{$\mathrm{TFF} 3, \mu \mathrm{g} / \mathrm{ml}$} & \multirow[t]{2}{*}{$\mathrm{p}$ trend } \\
\hline & $<0.20$ & $0.20-0.50$ & $0.51-0.96$ & $\geq 0.97$ & \\
\hline Age, years & $65.1(5.4)$ & $64.3(6.0)$ & $65.5(5.4)$ & $63.9(5.5)$ & 0.41 \\
\hline Female, \% & 75.9 & 45.6 & 46.8 & 51.9 & 0.02 \\
\hline Black, \% & 10.3 & 13.2 & 34.2 & 27.2 & 0.002 \\
\hline Systolic blood pressure, mm Hg & $125.8(15.6)$ & $131.6(21.0)$ & $134.8(20.6)$ & $130.3(17.7)$ & 0.16 \\
\hline Antihypertensive medication use, $\%$ & 29.3 & 33.8 & 39.2 & 48.1 & 0.02 \\
\hline Diabetes, $\%$ & 5.2 & 16.2 & 13.9 & 27.2 & 0.002 \\
\hline BMI & $28.4(6.1)$ & $29.2(5.3)$ & $27.5(4.5)$ & $28.3(4.8)$ & 0.46 \\
\hline Current smoking, $\%$ & 13.8 & 7.4 & 12.7 & 18.8 & 0.21 \\
\hline High-density lipoprotein cholesterol, mg/dl & $53.0(22.4)$ & $47.0(14.6)$ & $53.3(9.9)$ & $49.7(15.2)$ & 0.79 \\
\hline Triglycerides, mg/dl & $138.9(69.9)$ & $146.3(71.4)$ & $132.6(98.7)$ & $148.9(78.9)$ & 0.69 \\
\hline Urinary ACR, mg/g & $4.0[2.7,7.5]$ & $2.4[1.3,6.4]$ & $3.1[1.1,7.4]$ & $3.5[1.2,7.1]$ & 0.15 \\
\hline Baseline eGFR, $\mathrm{ml} / \mathrm{min} / 1.73 \mathrm{~m}^{2}$ & $79.2(11.5)$ & $84.3(17.4)$ & $82.1(13.3)$ & $83.5(14.9)$ & 0.23 \\
\hline Follow-up eGFR, $\mathrm{ml} / \mathrm{min} / 1.73 \mathrm{~m}^{2}$ & $68.5(18.7)$ & $64.8(19.2)$ & $63.3(21.7)$ & $62.9(25.9)$ & 0.13 \\
\hline Change in eGFR, $\mathrm{ml} / \mathrm{min} / 1.73 \mathrm{~m}^{2}$ & $-10.7(19.9)$ & $-19.5(21.2)$ & $-18.8(22.9)$ & $-20.6(25.7)$ & 0.03 \\
\hline
\end{tabular}

Continuous characteristics are presented as mean (SD) except urinary ACR, which is presented as median [25th, 75th percentile].

Table 3. OR (95\% CI) for the association between TFF3 quartile and incident CKD in the ARIC Study

\begin{tabular}{|c|c|c|c|c|c|}
\hline & \multicolumn{4}{|l|}{$\mathrm{TFF} 3, \mu \mathrm{g} / \mathrm{ml}$} & \multirow[t]{2}{*}{$\mathrm{p}$ trend } \\
\hline & $<0.20$ & $0.20-0.50$ & $0.51-0.96$ & $\geq 0.97$ & \\
\hline Cases/total, n & $21 / 58$ & $33 / 68$ & $43 / 79$ & $46 / 81$ & \\
\hline Unadjusted & 1.00 (reference) & $1.84(0.80,4.22)$ & $2.43(1.06,5.53)$ & $2.77(1.22,6.28)$ & 0.02 \\
\hline Model $1^{\mathrm{a}}$ & 1.00 (reference) & $1.91(0.83,4.42)$ & $2.47(1.08,5.65)$ & $2.87(1.25,6.55)$ & 0.01 \\
\hline Model 1 + urinary ACR & 1.00 (reference) & $1.83(0.76,4.40)$ & $2.66(1.10,6.43)$ & $2.90(1.20,6.97)$ & 0.02 \\
\hline
\end{tabular}

a Adjusted for diabetes, systolic blood pressure, antihypertensive medication use, current smoking, BMI, high-density lipoprotein cholesterol, triglycerides, and baseline eGFR.

The continuous relationship between TFF3 and incident CKD is shown in figure 2. Higher TFF3 was associated with a greater probability of being a case in a loglinear manner ( $\mathrm{p}=0.25$ for deviation from linearity). Twofold higher TFF3 was associated with a 29\% (95\%CI: $8 \%, 54 \%$ ) higher odds of developing incident CKD (table 4). As for the categorical analyses, adjustment for covariates strengthened the association slightly. The OD was similar in a model adjusting for diabetes, current smoking, BMI, high-density lipoprotein cholesterol, triglycerides, and eGFR (OR = 1.35; 95\% CI: 1.10, 1.63). Further adjustment for log ACR did not change the OR ( $\mathrm{p}=$ $0.003)$.

The association between the ratio of urinary TFF3 to urinary creatinine and incident CKD were somewhat weaker. After full adjustment, each twofold higher urinary TFF3:creatinine was associated with an adjusted OR of 1.22\% (95\% CI: 0.97, 1.53\%) for incident CKD.

Twofold higher TFF3 was significantly associated with incident CKD in analyses limited to males (adjusted $\mathrm{OR}=1.45 ; 95 \%$ CI: $1.03,2.04$ ) or females (adjusted $\mathrm{OR}=1.33 ; 95 \% \mathrm{CI}: 1.02,1.75)$. The OR associated with twofold higher TFF3 did not differ significantly between Whites (adjusted OR $=1.38$; 95\% CI: 1.11, 1.72) and Blacks (adjusted OR $=1.71 ; 95 \% \mathrm{CI}$ : 0.78, 3.73), or between those with diabetes (adjusted OR $=1.29 ; 95 \%$ CI: $1.04,1.60$ ) and those without diabetes (adjusted $\mathrm{OR}=1.07$; 95\% CI: $0.43,2.64$ ), though power was limited for these analyses. 


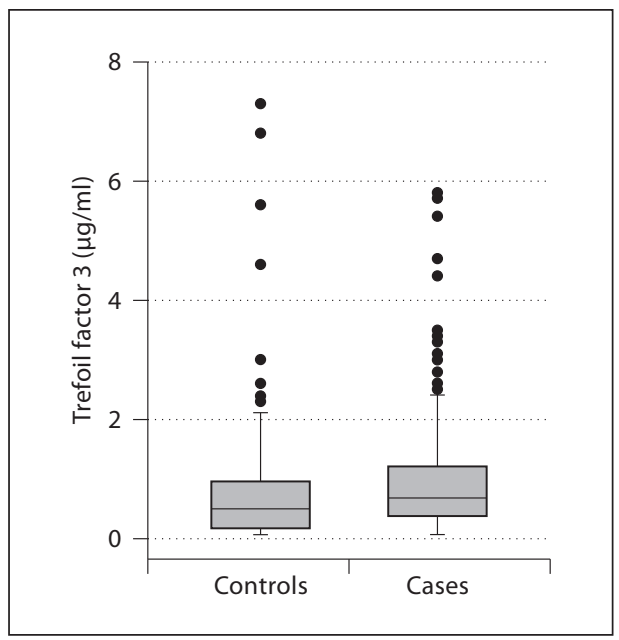

Fig. 1. Box plot of urinary TFF3 concentration, by case status.

Table 4. OR (95\% CI) for the association between twofold higher TFF3 and incident CKD in the ARIC Study

\begin{tabular}{lll}
\hline & OR $(95 \%$ CI $)$ & p value \\
\hline Unadjusted & $1.29(1.08,1.54)$ & 0.004 \\
Model 1 & $1.34(1.10,1.63)$ & 0.003 \\
Model 1 + urinary ACR & $1.35(1.11,1.64)$ & 0.003 \\
\hline
\end{tabular}

a Adjusted for diabetes, systolic blood pressure, antihypertensive medication use, current smoking, BMI, high-density lipoprotein cholesterol, triglycerides, and baseline eGFR.

\section{Discussion}

In this matched case-control study, higher TFF3 was strongly associated with incident CKD over a median follow-up of 8.6 years. Baseline TFF3 levels were higher among Blacks, females, individuals with diabetes and those using antihypertensive medications. Higher TFF3 remained significantly associated with incident CKD after adjustment for these factors, as well as for other CKD risk factors, including urinary albumin excretion.

TFF3 is a member of the trefoil factor family (TFF; consisting of TFF 1, TFF 2 and TFF 3), peptides which are secretory products of a variety of mucin-producing epithelial cells, including the gastrointestinal tract [22]. These peptides play a crucial role in the defense and re-

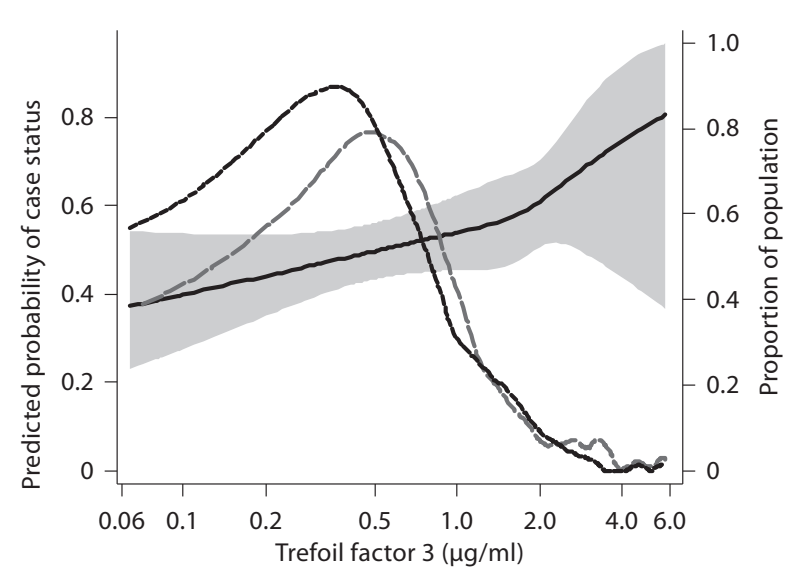

Fig. 2. Predicted probability of case status, by urinary TFF3 concentration. Shaded area represents 95\% CI. Dashed lines represent distribution of TFF3 concentration among controls (short dashes) and cases (long dashes).

pair of the mucosa through numerous mechanisms, including formation of mucous barriers, rapid repair by cell migration ('restitution'), antiapoptopic effects, and modulation of differentiation and immune response and inflammatory processes [23]. Pathologic expression of TFF peptides has been observed during a variety of chronic inflammatory diseases and certain tumors [24, 25]. TFF3 has been accepted by the FDA and the EMEA as a biomarker of drug-induced acute kidney tubular alterations in rat studies used to support clinical trials [13]. This decision was based on changes in urine TFF3 levels in rats with 2 weeks of drug-induced nephrotoxicity.

Recent experiments in humans have found TFFs synthesized throughout the urinary tract, with TFF3 being the predominate TFF in cells of the proximal and distal tubules and collecting duct [26]. Synthesis of TFF3 was found primarily in the basal and intermediate layers. The authors hypothesized that TFF3 may be partly responsible for the regenerative capacity of the kidney, possibly through restitution, which is initiated very quickly after injury [27-29], and/or effects on differentiation, which takes place over a longer time period [30-32]. Others have hypothesized similar roles for TFF3 in the gastric antrum $[32,33]$.

The role of TFF3 in CKD is unknown. We speculate that TFF3 is involved in the repair of injury, presumably to the tubular epithelium, and that the higher levels observed in the urine are indicative of ongoing repair, 
which, in turn, is indicative of damage or ongoing inflammatory processes. If this hypothesis is correct, TFF3 may be useful as a marker of CKD risk. Levels of TFF3 were much higher in participants with diabetes and in participants using antihypertensive medications than in their counterparts. The reasons for these differences are unknown. Diabetes and hypertension are potent risk factors for CKD [18], and these differences may provide additional evidence that TFF3 levels are associated with ongoing kidney damage. The lack of an association between TFF3 and urinary ACR argues against this hypothesis, but the restricted range of ACR values in these studies limits our ability to fully explore this potential association. The reasons for higher levels among Blacks compared to Whites and women compared to men are unknown.

This study has several limitations, including the use of a case-control design, which excludes individuals that died or were lost to follow-up or were ineligible for the follow-up examination. Any systematic bias, however, would likely attenuate the observed association. The relatively small sample size provided limited power for investigating associations within various subgroups, such as by diabetes status. The relative small sample size does not preclude statistical chance as an explanation for this difference. The definition of incident CKD (i.e., follow- up eGFR $<60 \mathrm{ml} / \mathrm{min} / 1.73 \mathrm{~m}^{2}$ and a $\geq 25 \%$ decrease in eGFR) was based on a single serum creatinine measurement at each visit, which can be imprecise. The case definition, therefore, may not detect significant changes in kidney function, or may detect clinically insignificant decreases $[18,19]$. Again, any imprecision would likely result in observing attenuated associations. This is a pilot study, and the observed associations should be explored in larger studies.

In conclusion, we found that higher urinary levels of TFF3 were associated with incident CKD. If confirmed in additional studies, TFF3 may be useful as a marker of future risk of CKD.

\section{Acknowledgements}

The Atherosclerosis Risk in Communities Study is carried out as a collaborative study supported by National Heart, Lung, and Blood Institute contracts N01-HC-55015, N01-HC-55016, N01HC-55018, N01-HC-55019, N01-HC-55020, N01-HC-55021, and N01-HC-55022 with the ARIC carotid MRI examination funded by U01HL075572-01. The ARIC sample assays were supported by the National Institute of Diabetes and Digestive and Kidney Diseases (1 R01 DK076770-01). The authors thank the staff and participants of the ARIC Study for their important contributions.

\section{References}

1 Astor BC, Hallan SI, Miller ER III, et al: Glomerular filtration rate, albuminuria, and risk of cardiovascular and all-cause mortality in the US population. Am J Epidemiol 2008; 167:1226-1234.

2 Shlipak MG, Sarnak MJ, Katz R, et al: Cystatin $\mathrm{C}$ and the risk of death and cardiovascular events among elderly persons. N Engl J Med 2005;352:2049-2060.

-3 Bash LD, Astor BC, Coresh J: Risk of incident ESRD: a comprehensive look at cardiovascular risk factors and 17 years of follow-up in the Atherosclerosis Risk in Communities (ARIC) Study. Am J Kidney Dis 2010;55:3141.

4 Fliser D, Kronenberg F, Kielstein JT, et al: Asymmetric dimethylarginine and progression of chronic kidney disease: the mild to moderate kidney disease study. J Am Soc Nephrol 2005; 16:2456-2461.

5 Fliser D, Kollerits B, Neyer U, et al: Fibroblast growth factor 23 (FGF23) predicts progression of chronic kidney disease: the Mild to Moderate Kidney Disease (MMKD) Study. J Am Soc Nephrol 2007;18:2600-2608.
-6 Spanaus KS, Kronenberg F, Ritz E, et al: B type natriuretic peptide concentrations predict the progression of nondiabetic chronic kidney disease: the Mild-to-Moderate Kidney Disease Study. Clin Chem 2007;53:12641272 .

-7 Kronenberg F: Emerging risk factors and markers of chronic kidney disease progression. Nat Rev Nephrol 2009;5:677-689.

8 Bleyer AJ, Shemanski LR, Burke GL, et al: Tobacco, hypertension, and vascular disease: risk factors for renal functional decline in an older population. Kidney Int 2000;57:20722079.

-9 Fox CS, Larson MG, Leip EP, et al: Predictors of new-onset kidney disease in a community-based population. JAMA 2004;291:844850.

10 Muntner P, Coresh J, Smith JC, et al: Plasma lipids and risk of developing renal dysfunction: the Atherosclerosis Risk in Communities Study. Kidney Int 2000;58:293-301.

11 James MT, Hemmelgarn BR, Tonelli M: Early recognition and prevention of chronic kidney disease. Lancet 2010;375:1296-1309.
12 Wright JT Jr, Bakris G, Greene T, et al: Effect of blood pressure lowering and antihypertensive drug class on progression of hypertensive kidney disease: results from the AASK trial. JAMA 2002;288:2421-2431.

13 Coons SJ: The FDA's critical path initiative: a brief introduction. Clin Ther 2009;31:25722573

14 Podolsky DK, Lynch-Devaney K, Stow JL, et al: Identification of human intestinal trefoil factor. Goblet cell-specific expression of a peptide targeted for apical secretion. J Biol Chem 1993;268:12230

15 Taupin D, Podolsky DK: Trefoil factors: initiators of mucosal healing. Nat Rev Mol Cell Biol 2003;4:721-732.

16 ARIC Investigators: The Atherosclerosis Risk in Communities (ARIC) Study: design and objectives. Am J Epidemiol 1989;129: 687-702.

17 Wasserman BA, Astor BC, Sharrett AR, et al: MRI measurements of carotid plaque in the Atherosclerosis Risk in Communities (ARIC) Study: methods, reliability and descriptive statistics. J Magn Reson Imaging 2010;31:406-415 
18 Bash LD, Coresh J, Kottgen A, et al: Defining incident chronic kidney disease in the research setting: The ARIC Study. Am J Epidemiol 2009;170:414-424.

-19 Coresh J, Astor BC, McQuillan G, et al: Calibration and random variation of the serum creatinine assay as critical elements of using equations to estimate glomerular filtration rate. Am J Kidney Dis 2002;39:920-929.

-20 Stevens LA, Coresh J, Feldman HI, et al: Evaluation of the modification of diet in renal disease study equation in a large diverse population. J Am Soc Nephrol 2007; 18:27492757.

-21 Papp AC, Hatzakis H, Bracey A, et al: ARIC hemostasis study. I. Development of a blood collection and processing system suitable for multicenter hemostatic studies. Thromb Haemost 1989;61:15-19.

-22 Hoffmann W: Trefoil factor family (TFF) peptides and chemokine receptors: a promising relationship. J Med Chem 2009;52:65056510 .
23 Hoffmann W: TFF (trefoil factor family) peptides; in Kastin AJ (ed): Handbook of Biologically Active Peptides. San Diego, Elsevier, 2006, pp 1147-1154.

24 Hoffmann W, Jagla W: Cell type specific expression of secretory TFF peptides: colocalization with mucins and synthesis in the brain. Int Rev Cytol 2002;213:147-181.

-25 Wong WM, Poulsom R, Wright NA: Trefoil peptides. Gut 1999;44:890-895.

26 Rinnert M, Hinz M, Buhtz P, et al: Synthesis and localization of trefoil factor family (TFF) peptides in the human urinary tract and TFF2 excretion into the urine. Cell Tissue Res 2010;339:639-647.

27 Hoffmann W: Trefoil factors TFF (trefoil fac tor family) peptide-triggered signals promoting mucosal restitution. Cell Mol Life Sci 2005;62:2932-2938.
28 Pawar S, Kartha S, Toback FG: Differential gene expression in migrating renal epithelial cells after wounding. J Cell Physiol 1995;165: 556-565.

29 Bonventre JV: Dedifferentiation and proliferation of surviving epithelial cells in acute renal failure. J Am Soc Nephrol 2003;14 (suppl 1):S55-S61.

30 Romih R, Koprivec D, Martincic DS, et al: Restoration of the rat urothelium after cyclophosphamide treatment. Cell Biol Int 2001; 25:531-537.

31 Veranic P, Romih R, Jezernik K: What determines differentiation of urothelial umbrella cells? Eur J Cell Biol 2004;83:27-34.

32 Hoffmann W: Regeneration of the gastric mucosa and its glands from stem cells. Curr Med Chem 2008;15:3133-3144.

$>33$ Kouznetsova I, Peitz U, Vieth M, et al: A gradient of TFF3 (trefoil factor family 3) peptide synthesis within the normal human gastric mucosa. Cell Tissue Res 2004;316:155-165. 